\title{
CROSS-SECTIONAL AREA MEASUREMENTS FOR TENDON SPECIMENS: A COMPARISON OF SEVERAL METHODS*
}

\author{
DONALD G. ELLIS \\ Department of Physical Medicine and Rehabilitation, The University of Michigan Medical \\ Center, Ann Arbor, Mich. 48104, U.S.A.
}

\begin{abstract}
Several methods for measuring cross-sectional areas of cat extensor digitorum communis tendons, were evaluated in terms of repeatability. Estimates of correction factors to be used in relating measurements by different methods were also obtained. The measurements examined included: (1) moist specimen weight per unit length, (2) moist specimen displacement volume per unit length, (3) dry specimen weight per unit length, (4) dry specimen displacement volume per unit length, (5) area measured with an area micrometer similar in principle to the instrument described by Walker et al. (1964), (6) area by shadow amplitude contour reconstruction, and (7) planimeter measurements on photomicrographs of histological sections. The results obtained suggested that dry specimen weight per unit length could be expected to yield the most repeatable single measurements for cross-sectional areas of tendon specimens. For measurements of fresh, moist specimens, gravimetric methods and measurements with the area micrometer were found roughly comparable in repeatability and better than shadow amplitude contour reconstruction. The area micrometer measurements seemed less sensitive to slight drying of the specimen, but were considerably influenced by the pressure applied to the specimen during measurement. Comparisons of the values for cross-sectional area obtained by the various methods showed significant systematic differences, with several pairs of methods giving measurements which differed by factors of 2 or more. Measurements by methods which were more or less local in nature demonstrated sizable regional variations in cross-sectional areas of the specimens even though they had a relatively uniform appearance.
\end{abstract}

\section{INTRODUCTION}

RESULTS from materials testing procedures are normally reported with the load applied to the test specimen expressed in terms of stress, or load per unit cross-sectional area. This permits the comparison of results from tests on specimens of different sizes and provides a basis for the prediction of the performance of various elements from data obtained with different sized elements of the same type of material. However, for such comparisons and predictions to be quantitatively meaningful, it is necessary that the cross-sectional areas used in stress computation be determined by methods which are reasonably accurate and consistent.

Several methods for determining specimen cross-sectional areas have been used by investigators studying the mechanical prop- erties of connective tissue. Rigby et al. (1959) used diameter measurements made with a microscope having a calibrated eyepiece micrometer and assumed circular crosssections to obtain cross-sectional areas of rat tail tendons. Gross width and thickness measurements made with mechanical instruments have been used for specimens which were assumed to have approximately rectangular cross-sections; fascia lata (Gratz, 1931), ligamentum flavum (Nunley, 1958), and plantar fascia (Wright and Rennels, 1964). Walker et al. (1964) developed an instrument for determining the cross-sectional areas of tendons by compressing the specimens to rectangular sections of known width and measuring the heights of the sections by means of a micrometer head. Cross-sectional areas based on specimen length and volume,

\footnotetext{
${ }^{*}$ Received 22 October 1968.
} 
determined from the weight and density of the moist specimen, have been used by VanBrocklin and Ellis (1965), Abrahams (1967), and Matthews and Ellis (1968), while Elden $(1963,1964 a, 1964 b)$ has used dry specimen weight per unit length as a measure of crosssection. Earlier, however, Cronkite (1936) had rejected methods based on specimen volumes determined either from weight and specific gravity or from liquid displacement because he found that these methods did not give consistent results. Instead, he cut sections $0.5-1.0 \mathrm{~mm}$ thick from his tendon specimens, projected shadows of these on a screen, made tracings of the projected sections, and measured the areas of these tracings with a planimeter. Ellis (1968) has described a method which uses a partial shadow of the specimen as the basis for constructing a surface contour which can be integrated to give specimen area. This method does not require sectioning of the specimen but assumes the contour of the cross-section be convex.

Comparisons of published data on the mechanical properties of connective tissue have tended to assume that the reported stresses could be compared directly even though they were computed using cross-sectional areas measured by these different methods. However, it seems likely that there exist real, systematic differences between these methods. For example, one might expect cross-sectional areas measured by the instrument of Walker et al. (1964) which compresses the specimen slightly to be consistently smaller than those measured by methods which do not, such as the gravimetric method employed by VanBrocklin and Ellis (1965) or the ocular micrometer used by Rigby et al. (1959). Considering the nature of crosssectional area measurement techniques which may be applied to connective tissue specimens, it is reasonable to assume as a first approximation that areas measured by one method tend to differ from those measured by another by a constant factor, $K$.
The work described in this paper was designed to obtain values for this constant, $K$, for pairs of methods which might be applied to the measurement of tendons having crosssectional areas on the order of $0.2 \mathrm{~mm}^{2}$ and to evaluate the repeatability of each of these methods. The measures examined were: (1) Moist specimen weight per unit length, (2) moist specimen displacement volume per unit length, (3) dry specimen weight per unit length, (4) dry specimen displacement volume per unit length, (5) micrometer measured area, using an instrument similar in principle to that described by Walker et al. (1964), (6) shadow amplitude contour reconstruction using the method described by Ellis (1968), and (7) planimeter measurements on photomicrographs of histological preparations.

\section{MATERIALS AND METHODS}

In order to compare these methods for determining the cross-sectional areas of tendon specimens, the area of each of 16 cat extensor digitorum communis tendons was determined twice by each method which could be applied to fresh, moist material and once by one of the two types of destructive methods which could not. The cross-sectional areas of 8 of the tendons, one from each animal, were measured by each of the types of destructive methods, dry specimen gravimetric and histological.

From the data so obtained the repeatability of each nondestructive method was evaluated by computing a correlation coefficient, $R$, for measurements by this method. For this computation the two measurements on a single specimen were taken as the coordinates of one point in the sample space.

A value for the factor $K$ relating each pair of methods was obtained by a least squares fit of the equation $y=K x$ to the collection of sample points each of whose coordinates were the values of cross-sectional area measured for one specimen by each of the two methods.

Correlation coefficients were computed 
for pairs of methods as well as for pairs of determinations by individual methods and provided additional measures of variability. In addition, values of $K$ were obtained for pairs of determinations by each method. These provided estimates of changes which might have occurred in the course of the measurement protocol.

The protocol for this study is described in detail later in this section after descriptions of the various area measurement procedures have been given.

\section{Area measurement procedures}

(a) Moist specimen gravimetric methods. The net weight of each specimen in air and the weight of distilled water which it displaced were determined using a Mettler $\mathrm{H} 16$ semimicro balance having an accuracy of \pm 0.02 $\mathrm{mg}$ over the range of specimen weights. When each specimen was to be weighed it was blotted lightly and draped over a small stainless steel hook weighing approximately $35 \mathrm{mg}$. This hook was then suspended from one of the series of hooks which suspend the balance pan; and the hook-specimen combination was weighed. Then, a cylinder of distilled water (carried on a rack-and-pinion positioning mechanism housed inside the balance enclosure) was raised so that the hookspecimen combination was immersed to the level of a small paint mark near the middle of the hook shank, and the hook-specimen combination was again weighed. After both weighings, the specimen was removed from the hook; the hook alone was weighed in air and immersed to the level of the paint mark; and these weights were subtracted from those previously obtained to yield specimen weight and displacement.

(b) Dry specimen gravimetric methods. For dry specimen gravimetric determinations, the specimen was draped over the stainless steel hook mentioned above and was dried under ambient conditions (temperature $20^{\circ}-25^{\circ} \mathrm{C}$, relative humidity 50-80 per cent) for 22-26 hr. The weighing steps described above were then carried out.

(c) Area micrometer method. The configuration of the area micrometer employed is indicated in Fig. 1. In this instrument the specimen was placed between a pair of stainless steel side blocks which were positioned

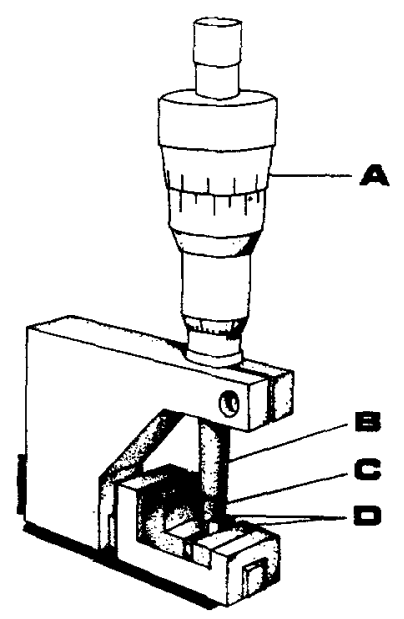

Fig. 1. Area micrometer; (A) Torque measuring thimble, (B) Spindle, (C) Plug, (D) Side blocks. Specimen was placed between blocks (D) and beneath blade portion of plug (C).

0.0307 in. apart on a rectangular stainless stainless steel bar which formed the micrometer anvil. A cylindrical stainless steel plug having at one end a rectangular blade 0.0305 in. thick by 0.173 in. wide was then placed with its blade between the side blocks and resting on the specimen. The thimble of the micrometer head (Starrett, 1463) was turned to bring the spindle into contact with the plug until the thimble torque, measured by a spring-type torque thimble mounted on the micrometer thimble, reached its prescribed value (see protocol). The thimble was then released and the micrometer read. With the slot and plug dimensions used and the micrometer head located so as to read zero, when the plug was in place and no specimen present, the measured area in square millimeters was 20 times the micrometer reading in inches. 
(d) Shadow amplitude contour reconstruction method. The principle of operation of the shadow amplitude method is indicated in Fig. 2. The specimen (seen in cross-section in Fig. 2a) is partially illuminated by a collimated rectangular light beam limited in such a way that light from the beam passes the specimen on one side only. The specimen intercepts a portion, $A$, of the beam which may be called the shadow amplitude. Note that

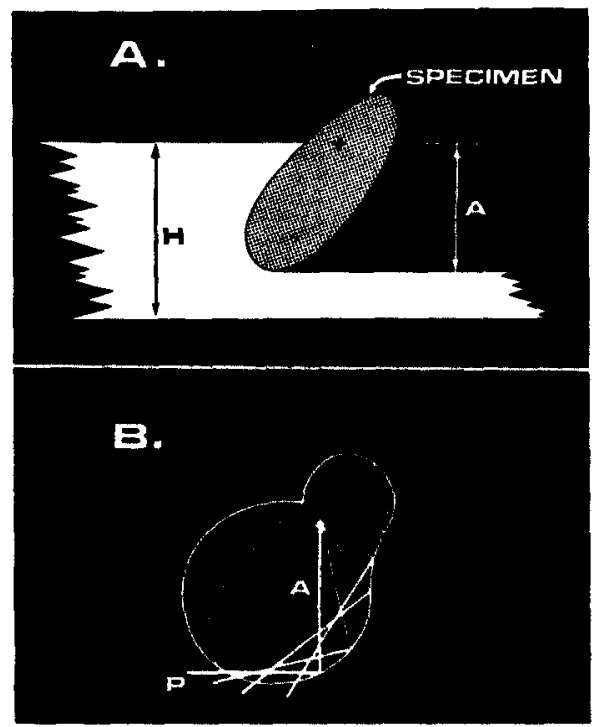

Fig. 2. Shadow amplitude contour reconstruction. (A) Measurement of shadow amplitude (Note position of specimen center of rotation.) (B) Reconstruction of specimen outline from a polar plot of shadow amplitude.

the shadow amplitude, $A$, is equal to the distance between the obstructed edge of the beam and a tangent to the specimen surface which is parallel to the beam. Now, let the specimen be rotated about an axis which satisfies the following conditions: (1) The axis lies on the projection of the obstructed edge of the light beam, (2) it lies within the specimen, and (3) it is perpendicular to the beam. The shadow amplitude, $A$, will be the distance from this axis to a tangent parallel to the beam for every angular position of the specimen.

Given the shadow amplitude, $A$, as a function of angular position, $\theta$, and assuming the specimen contour to be convex, it is possible to obtain the specimen contour by reconstructing this tangent for each angular position and taking the specimen contour as the outer boundary of the area enclosed by the collection of tangents. This can be done on a polar plot of $A$ as a function of $\theta$ as shown in Fig.2b. For any radius vector, the corresponding tangent is the perpendicular, $P$, to the radius vector at its point of intersection with the shadow amplitude plot.

The equipment used to obtain polar plots of $A$ as a function of $\theta$ is indicated diagrammatically in Fig. 3. Here the light passed by the specimen was detected by a phototransistor circuit, and a signal corresponding to the output obtained with an unobstructed

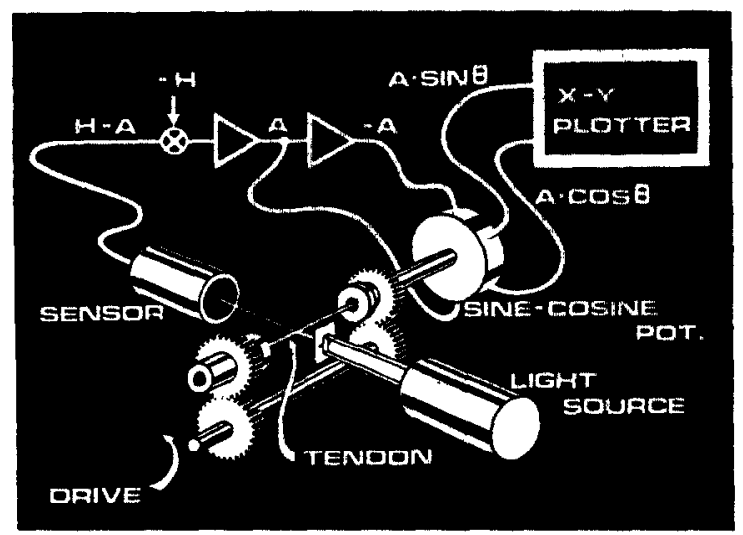

Fig. 3. System used to obtain polar shadow amplitude plots for tendon specimens.

beam, $H$, was added to the detector output to give a signal proportional to the shadow amplitude, $A$. This signal was amplified, inverted, and used as excitation for a sine-cosine potentiometer connected to one of the spindles which rotated the specimen. The outputs of the potentiometer, $A \sin \theta$ and $A \cos \theta$, were then recorded on a Data Equipment Company Plotmatic $600 \mathrm{X}-\mathrm{Y}$ plotter to give polar shadow amplitude plots from which contours were reconstructed graphically, as indicated in Fig. 2b. Scaling of the plots $\left(10 \mathrm{in}^{2}\right.$ to the $\left.\mathrm{mm}^{2}\right)$ was such that planimeter measurements of the reconstructed contours were easily reproducible to within 
\pm 1 per cent. In this apparatus the specimen was gripped by a pair of 2 -jaw collets each magnetically drawn into a tapered spindle bore. Each spindle ran on two lightly preloaded precision-5 ball bearings (New Departure SS.Z.99.R-6.BBZH5738) and was driven by an anti-backlash instrument gear on the drive shaft. Calibration with stainless steel bars of known diameter showed the total uncertainty for axis of rotation and slit position to have been less than \pm 0.0003 in. (uncertainty in measured area approximately \pm 5 per cent for specimens in the size range of the experimental material).

For each measurement on an experimental specimen the offset signal $[H]$ was first adjusted so that the output was zero with the unobstructed beam. The lamp was then turned off, and the amplifier gains adjusted, if necessary, to give a circular trace with a diameter corresponding to twice the slit width. The lamp was then turned on again and the (zero) output checked. After this initial calibration, a standard bar of $1.00 \mathrm{~mm}^{2}$ crosssection was placed in the instrument and its shadow amplitude plot obtained. Then, the specimen was mounted and its plot obtained. Several rotations of the specimen were employed for each plot. After the specimen plot had been obtained the specimen contour was reconstructed graphically, and the areas of the 'light off circle, the calibration bar circle, and the specimen contour were measured with a polar planimeter.

(e) Histological methods. Each specimen for which measurements were to be made on histological sections was cut into two portions. One portion was placed in Ringer's solution and sent to an outside laboratory for paraffin embedding and sectioning. The other portion was suspended between the ends of a specially designed fluorocarbon boat, dehydrated through a graded series of ethanol solutions and embedded in nitrocellulose. The paraffin preparations were stained with hematoxylin and eosin while the nitrocellulose preparations were stained with
Van Gieson's stain. Several sections were cut from each paraffin-embedded portion, and one section which appeared to have been least distorted by the preparation procedures was selected for measurement. Two sections spaced approximately $1 \mathrm{~cm}$ apart were cut from each nitrocellulose embedded portion; and each section was measured. Measurements were made using a polar planimeter on photomicrographs with a total magnification of $80 \times$.

\section{Experimental protocol}

Each cat was sacrificed with intraperitoneal sodium pentobarbital. All eight of its extensor digitorum communis tendons were then dissected from the front paws and placed in a tray of Ringer's solution. From among these tendons, the first tendon to be measured was selected and was cut to a length of $4.00 \mathrm{~cm}$ using the cutting machine illustrated in Fig. 4. In this machine two swinging arms are moved toward the specimen by links connected to a spring-loaded sliding post. Each arm carries a rubber coated pad which slides along the 'chopping block' surface so that the tendon is straightened and stretched slightly by the pads before the blades, also carried one on each arm, strike the tendon cutting it to the prescribed length.

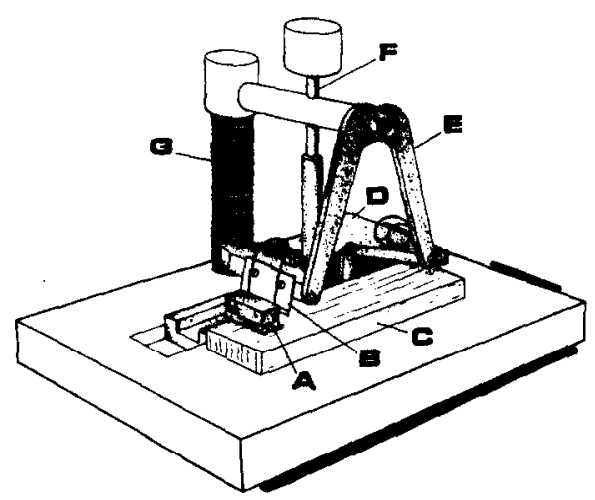

Fig. 4. Tendon cutting machine; (A) Tendon straightening pad, (B) Blade, (C) Chopping block, (D) Arm carrying pad and blade, (E) Linkage to arm, (F) Guide pin, (G) Spring-loaded sliding post. (See text for description of operation.) 
After cutting, one end of the specimen was marked with a small ink spot for identification.

Then, the specimen was measured twice by each of the nondestructive techniques. The sequence of measurements was always as indicated in Fig. 5, the starting point in the sequence was permuted one step (one place around the ring) for each new specimen tested. With the specimens from 4 of the animals, the first measurement by the shadow amplitude contour reconstruction method was taken at about $1 \mathrm{~cm}$ from the marked end and the second at a similar distance from the unmarked end; with tendons from the other 4 animals both measurements were made about $1 \mathrm{~cm}$ from the marked end. Each 'measurement' with the area micrometer consisted of 6 area determinations, 3 taken approximately 1 $\mathrm{cm}$ from the marked end of the specimen and 3 taken approximately $1 \mathrm{~cm}$ from the unmarked end. In each set of three, the first was made at a thimble torque of $0.01 \mathrm{Mdyn}-\mathrm{cm}$, the second at a thimble torque of $0.02 \mathrm{Mdyn}-\mathrm{cm}$, and the third at a thimble torque of $0.04 \mathrm{Mdyn}-\mathrm{cm}^{*}$.

After all nondestructive measurements were made on the first specimen from a cat, the specimen was cut into two portions which were subsequently prepared for histological examination.

Then, the second tendon to be measured was selected, cut, and carried through the sequence of nondestructive measurements described above. When these measurements were completed, desiccation of this specimen for dry specimen gravimetric measurements was begun.

All nondestructive measurements on the two specimens from each cat were carried out within $2 \frac{1}{2} \mathrm{hr}$ after the animal's death.

\section{RESULTS}

\section{Repeatability}

Analysis of the data with paired measure-

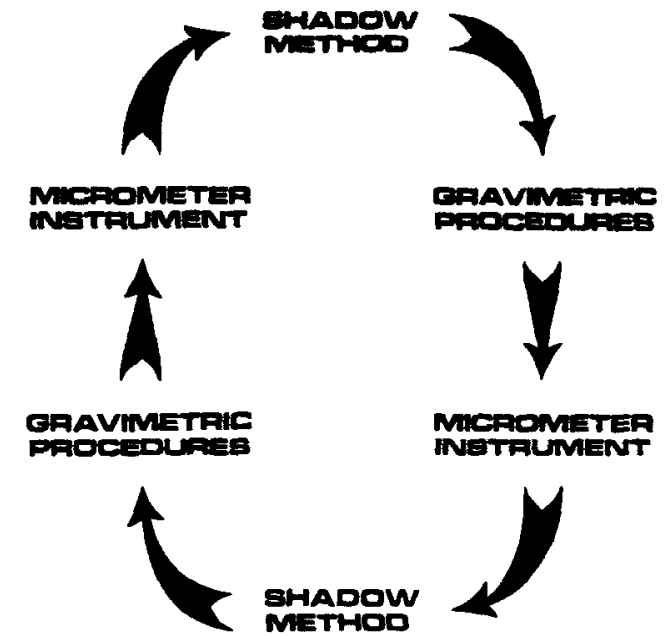

Fig. 5. Sequence of nondestructive measurement procedures. Starting point was shifted one place around the ring for each new specimen measured.

ments by the same method as coordinates in the sample space provides an indication of repeatability. For those methods not giving an average area over the full length of the specimen, only measurements taken at corresponding locations were included in this analysis. Thus, the shadow amplitude contour reconstruction data from only half of the specimens are included, while the area micrometer data has provided two sample points (one for each end) for each specimen at each thimble torque.

The correlation coefficients for repeatability of the nondestructive methods are presented in Table 1 along with values of the factor $K$ and the number of sample pairs in the analysis for each method.

These statistics indicate that, of the three types of nondestructive measurements, the moist specimen gravimetric measures showed the best correlation between first and second measurements while the area micrometer measurements showed the smallest systematic change between measurements ( $K$ close to 1$)$. The correlation between first, and second

*The corresponding pressures may be estimated by assuming the spindle thrust for each torque to have been the spindle end thrust for which that torque is the thimble breakaway torque and by taking the area of the end of the blade as the area over which this force acted. Based on measurements of thimble breakaway torque at a number of known spindle end forces for the micrometer head, the pressures corresponding to the thimble torques employed in the protocol would have been, roughly: $0 \cdot 7,2 \cdot 3$, and $5 \cdot 2 \mathrm{Mdyn} / \mathrm{cm}^{2}$. 
Table 1. Repeatability: correlation coefficients and slopes of the least squares lines $y=K x$, for the second measurement by each nondestructive method as a function of the first measurement by the same method, at the corresponding location

\begin{tabular}{|c|c|c|c|}
\hline Method & Correlation coefficient, $R$ & slope, $K$ & Number of sample pairs \\
\hline Shadow contour reconstruction & 0.740 & 0.875 & 8 \\
\hline $\begin{array}{l}\text { Thimble torque: } 0.01 \\
\text { Mdyn-cm } \\
\text { Mdyn-cm } \\
\text { Mimble torque: } 0.02 \\
\text { Thimble torque: } 0.04 \\
\text { Mdyn-cm }\end{array}$ & $\begin{array}{l}0.737 \\
0.834 \\
0.878\end{array}$ & $\begin{array}{l}0.958 \\
1.019 \\
1.001\end{array}$ & $\begin{array}{l}31 \\
32 \\
32\end{array}$ \\
\hline $\begin{array}{l}\text { Moist specimen displacement } \\
\text { volume per unit length } \\
\text { Moist specimen weight } \\
\text { per unit length }\end{array}$ & $\begin{array}{l}0.918 \\
0.922\end{array}$ & $\begin{array}{l}0.941 \\
0.935\end{array}$ & 16 \\
\hline
\end{tabular}

measurements with the area micrometer was found to increase with increasing thimble torque over the range of thimble torques used. The shadow amplitude contour reconstruction method gave both the lowest correlation between first and second measurements and the greatest systematic change in measurement $(K=0.875)$ of the three methods.

It was not possible to assess the repeatability of the dry-specimen gravimetric measures or the histological methods in the same way that the repeatability of the other methods was assessed, since all of the steps in these measurement procedures could not be repeated on a single specimen. An attempt was made, however, to examine the repeatability of the dry weight measurement for specimens which were dried only once. For this purpose, 8 specimens from 7 of the 8 cats were used. These specimens had been preserved by freezing. They were thawed, cut to $4.00 \mathrm{~cm}$ lengths, dried, and weighed in air, as previously described. They were subsequently reweighed 24 and $48 \mathrm{hr}$ after the initial weighing. Values of $R$ and $K$ were computed for pairs of weighings: For the second as a function of the first: $R=0.977$, $K=0.997$; for the third as a function of the first: $R=0.977, K=0.998$; and for the third as a function of the second: $R=0.9998$, $K=1.000$.

\section{Regional variations}

Three of the types of measurements examined, area micrometer measurements, shadow amplitude contour reconstructions, and measurements on histological preparations, gave cross-sectional area measurements which were more or less local in character and could be used to evaluate regional variations in the cross-sectional areas of the tendons used in the study. This evaluation was carried out using the same type of analysis previously described, i.e. computation of the correlation coefficient and slope of the least squares straight line through the origin. For the evaluation of regional variations in the specimens the paired data consisted of measurements made by the same method at distinctly separated locations on each specimen. In this analysis only area micrometer data obtained at a thimble torque of 0.04 Mdyn-cm (most repeatable condition) were used. The two measurements at each site were averaged giving one sample point for each of 16 specimens. Shadow amplitude contour reconstruction and histological sections from nitrocellulose embedded specimens each gave one sample point for each of 8 secimens. The computed correlation coefficients and slopes are presented in Table 2 along with the separation of measurement sites, number of sample pairs, and the length 
Table 2. Variation in cross-sectional area measurements at distinctly separated sites: correlation coefficients and slopes of least squares fitted lines $y=K x$

\begin{tabular}{lccccc}
\hline \multicolumn{1}{c}{ Measure } & $\begin{array}{c}\text { Correlation } \\
\text { coefficient, } R\end{array}$ & Slope, $K$ & $\begin{array}{c}\text { Separation } \\
\text { of sites, } \mathrm{cm}\end{array}$ & $\begin{array}{c}\text { Length over which } \\
\text { area was measured }\end{array}$ & $\begin{array}{c}\text { Number of } \\
\text { sample pairs }\end{array}$ \\
\hline $\begin{array}{l}\text { Area Micrometer: } \\
\begin{array}{l}\text { 0.04 Mdyn-cm } \\
\text { Thimble torque } \\
\text { (average of two measurements) }\end{array}\end{array}$ & 0.730 & 0.998 & 2 & $4.4 \mathrm{~mm}$ & 16 \\
$\begin{array}{l}\text { Shadow amplitude } \\
\text { contour reconstruction }\end{array}$ & 0.002 & 0.821 & 2 & $1.4 \mathrm{~mm}$ & 8 \\
$\begin{array}{l}\text { Nitrocellulose embedded } \\
\text { histological preparations }\end{array}$ & 0.463 & 0.971 & 1 & $\begin{array}{c}\text { roughly } \\
100 \mu\end{array}$ & 8 \\
\hline
\end{tabular}

of specimen over which each method 'averaged' the area (width of blade for micrometer instrument, width of light beam for shadow method, and thickness of sections for histological method).

For all three techniques, low correlation coefficients were obtained. For the two measures for which repeatability assessments were made, these correlation coefficients were significantly lower than the correlation coefficients for repeatability. Thus, it is unlikely that the size of the correlation coefficients can be accounted for on the basis of random errors in the methods, and variations in specimen size approaching the range of specimen sizes likely.

\section{Relation between methods}

For the analysis of the relations between cross-sectional area measurements by the various methods examined, all measurements on a specimen by a single method were averaged. This average was used as the measurement for that specimen by that method, and methods of measurements were taken in pairs for calculation of correlation coefficients and slopes of the least squares fit straight lines through the origin (correction factors for comparing tests which have used different measurements of cross-sectional area). Table 3 shows these statistics for pairs of measures examined in the study.

These statistics show sizable variations among cross-sectional areas measured by the different methods examined. For example, moist specimen displacement volumes per unit length tended to be about 3 times as large as the same quantities for the dry specimens, approximately 2.7 times the areas measured on sections from nitrocellulose embedded specimens, and, around 2.4 times those measured with the area micrometer, but less than 1.4 times the areas measured on sections from paraffin embedded specimens or by the shadow amplitude contour reconstruction method. Except for the case where the two moist specimen gravimetric measures, which were not independent, were paired, the correlation coefficients tended to be lower than those for repeatability and showed sizable variations among the pairs of measures. Some patterns in the distribution of correlation coefficient may be seen. For example, the correlations between the moist specimen measurements and the dry specimen weight per unit length tended to be relatively high. These patterns will be discussed in the following section.

In the above analysis, only those micrometer area instrument measurements made at a thimble torque of $0.04 \mathrm{Mdyn}-\mathrm{cm}$ were used. To evaluate the influence of thimble torque on the cross-sectional areas measured with this instrument, an analysis was performed in which the average of all measurements made on a specimen at thimble torque of $0.04 \mathrm{Mdyn}-\mathrm{cm}$ was paired with the average of all measurements made on the same 
CROSS-SECTIONAL ARIEA MEASUREMENTS

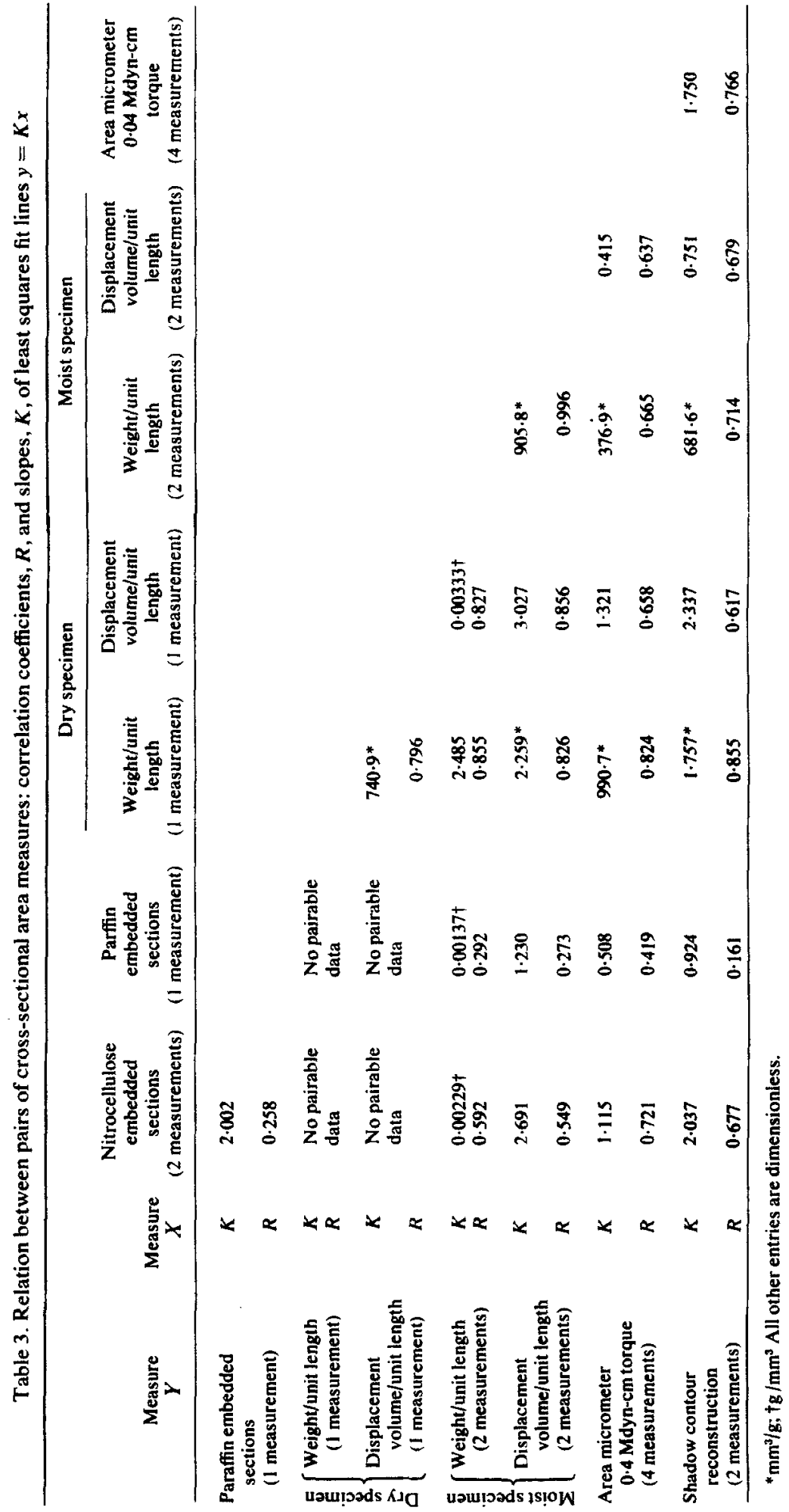


specimen at a thimble torque of $0.01 \mathrm{Mdyn}$ $\mathrm{cm}$ and with the average of all measurements on that specimen made at a thimble torque of $0.02 \mathrm{Mdyn}-\mathrm{cm}$. The values obtained for $K$, the slopes of the least squares lines $y=K x$, were 1.403 for measurements at 0.01 Mdyn$\mathrm{cm}$ thimble torque as a function of those at 0.04 Mdyn-cm and 1.196 for measurements at $0.02 \mathrm{Mdyn}-\mathrm{cm}$ as a function of measurements at 0.04 Mdyn-cm. The corresponding correlation coefficients were 0.923 and 0.961 , respectively. Thus, it is apparent that measurements obtained with this instrument were quite dependent on thimble torque, the measured area decreasing as the thimble torque (or pressure on the specimen) was increased.

\section{DISCUSSION}

To a large extent the present study has considered cross-sectional areas for tendon specimens determined from single measurements. It has been concerned mainly with two questions about possible methods which might be applied: (1) Which measures are likely to give the most repeatable results? and, (2) what adjustments need to be made when comparing data obtained using two different measurements?

The correlation coefficients computed for first and second measurements by those methods which were applicable to fresh, moist specimens showed these methods to have yielded relatively poor repeatability. Among these methods, the shadow amplitude contour reconstruction method was least repeatable, while moist specimen gravimetric measurements and measurements with the area micrometer were significantly more repeatable. Slopes of the least squares fit lines $y=K x$ for the moist specimen gravimetric methods showed a tendency for the measured area to decrease between the first and second measurement (possibly due to some drying of the specimen) while measurements by the area micrometer demonstrated little or no systematic change of this type.
The repeatability of measurements with the area micrometer increased with increasing thimble torque, probably as a result of two effects: (1) The higher pressures at higher thimble torques would have tended to force the specimen to conform better to the rectangular space which was measured; and (2) the variability in the spindle force between measurements was probably a smaller fraction of the total spindle force at higher thimble torques, an effect which was noted in the measurements which were made to determine thimble breakaway torque as a function of spindle end thrust. It might therefore be possible to select a thimble torque, or preferably a blade pressure, for which a micrometer instrument of this type would give correlation coefficients for repeatability at least as high as those obtained for the moist specimen gravimetric methods. If this were done, an area micrometer might provide the best method for situations where measurements of the cross-sectional areas of fresh, moist specimens are required, since this method seems to be insensitive to slight drying of the specimen and since it is extremely easy to apply. However, because of the dependence of measured area on blade pressure (thimble torque), it would probably be good practice to report the blade pressure used in any study where such an instrument is employed.

The repeatability of techniques involving desiccation or histological preparations was not evaluated in the same way as the repeatability of measurements on the fresh, moist specimens since it was not practical to repeat all of the steps in these procedures on single specimens. However, some assessment of the repeatability of the dry specimen gravimetric measurements and of the measurements on histological preparations can be made from the correlation coefficients for pairs of measurements by different methods. In general, if two techniques, $\mathrm{A}$ and $\mathrm{B}$, have relatively large random errors while a third technique, $\mathrm{C}$, is subject only to small random 
errors, and if all three techniques tend to measure the same quantity, then the correlation coefficients relating measurements by methods $A$ and $C$ and by methods $B$ and $C$ will tend to be larger than that relating $A$ and $B$. Conversely, if the random errors in $C$ are greater than those in $\mathrm{A}$ or $\mathrm{B}$, correlation coefficients between $A$ and $C$ and between $B$ and $C$ will tend to be smaller than that for $A$ and $B$.

The correlation coefficients for measurements on histological sections paired with other measurements (Table 3) were generally less than those for other measurement pairs. Thus, it would appear that measurements on histological sections tended to be highly variable measures of cross-sectional area. The use of an average of the measurements on two sections cut from each nitrocelluloseembedded specimen, as compared with only one section cut from each paraffin-embedded specimen, could account, at least in part, for the higher correlations between nitrocellulose preparations and other methods than between paraffin preparations and other methods.

In contrast, the correlation coefficients for dry specimen gravimetric measurements, especially dry specimen weight per unit length, paired with other measurements tended to be higher than those for other pairs of measurements. This would suggest that the dry specimen weight per unit length was probably the least variable of the techniques examined. The very good repeatability found for the dry weight determination further demonstrates this for one step in the measurement procedure.

From the values of the slopes of the least squares lines $y=K x$ fitted to measurements by various pairs of methods, it is apparent that there were sizable differences, up to a factor of approximately 3 , in the numerical values of the areas obtained by different methods. It might be interesting to consider the extent to which these differences are reflected in stress values reported in the literature. A particularly good comparison can be made between the results reported by Van
Brocklin and Ellis (1965) and those reported by Benedict et al. (1968). Both reports presented stress-strain curves along with elastic modulus data for fresh human toe extensor tendons. Benedict $e t$ al. included tendons of the extensor digitorum longus, extensor digitorum brevis, and extensor hallucis longus muscles in the group of extensor tendons which they examined while the work of VanBrocklin and Ellis dealt with the tendons of the extensor digitorum muscles. VanBrocklin and Ellis employed a moist specimen gravimetric method for determining crosssectional areas, while Benedict et al. used a micrometer area instrument. Stress-strain curves were obtained by VanBrocklin and Ellis for various stress application rates and covered only a range of relatively small stresses while Benedict et al. applied stresses up to the breaking strength of the material and used only one relatively low strain rate. The curves obtained by VanBrocklin and Ellis showed an initial increase in stiffness with increasing load while those obtained by Benedict et al. showed little of this effect. Thus it would probably be reasonable to compare the 'initial' value of elastic modulus from the study by Benedict et al. $(225,000 \mathrm{psi}$, or approximately $15,500 \mathrm{Mdyn} / \mathrm{cm}^{2}$ for extensor tendons) with the 'mean low rate elastic modulus' near the upper end of the stress range investigated by VanBrocklin and Ellis (approximately $5100 \mathrm{Mdyn} / \mathrm{cm}^{2}$ at a stress of $80 \mathrm{Mdyn} / \mathrm{cm}^{2}$ ). The ratio of these moduli is 0.33 , as compared to 0.415 which was the $K$ value obtained here for area micrometer measurements at a thimble torque of 0.04 Mdyn-cm as a function of moist specimen displacement volume per unit length. This suggests that much of the difference between moduli reported in these two reports might be accounted for by the differences in the cross-sectional area measurements employed.

Measurements by those methods which did not average over the full length of the specimen indicated the presence of significant regional variations in the cross-sectional areas 
of the cat extensor digitorum communis tendons, even though these specimens appeared relatively uniform in size. It is likely that investigators will begin to take such regional variations into account as studies on the mechanical properties of connective tissue become more sophisticated. This will require the development of repeatable techniques for measuring local cross-sectional areas, ultimately on the fresh, moist specimen while it is being subjected to mechanical testing procedures. Of the measurement techniques investigated here, only the shadow amplitude contour reconstruction method could be adapted for such measurements. The calibration of the instrument used in this study indicates that reasonable accuracy can be obtained with this procedure. However, the poor repeatability for measurements on tendon specimens (some of which may be accounted for by differences in the regions scanned at the 'same end' of the specimen and by specimen drying) suggests the need for procedural as well as hardware refinements.

Acknowledgement - This study was conducted as part of the University of Michigan Orthetics Research Project which receives partial support from funds granted by Health Education and Welfare Social and Rehabilitation Services.

\section{REFERENCRS}

Abrahams, M. (1967) Mechanical behavior of tendon in vitro. Med. \& Biol Engng 5, 433-443.

Benedict, J. V., Walker, L. B. and Harris, E. H. (1968) Stress-strain characteristics and tensile strength of unembalmed human tendon. J. Biomechanics 1, 53-63.

Cronkite, A. E. (1936) The tensile strength of human tendons. A nat. Rec. 64, 173-186.

Elden, H. R. (1963) The interaction of connective tissue with aqueous area. I. Reversible and irreversible effects. Biochim. biophys. Acta 75, 35-47.

Elden, H. R. (1964a) Aging of rat tail tendons. J. Geront. 19, 173-178.

Elden, H. R. (1964b) Hydration of connective tissue and tendon elasticity. Biochim. biophys. Acta 79, 592-599.

Ellis, D. G. (1968) A shadow amplitude method for measuring cross-sectional areas of biological specimens. 21 st A. Conf. on Engng in Med. \& Biol. Houston, Texas.

Gratz, C. M. (1931) Tensile strength and elasticity tests on human fascia lata. J. Bone Jt Surg. 13, 334-340.

Matthews, L. S. and Ellis, D. (1968) Viscoelastic properties of cat tendon: effects of time after death and preservation by freezing. J. Biomechanics 1, 65-71.

Nunley, R. L. (1958) The ligamenta flava of the dog. A study of tensile and physical properties. Am. J. phys. Med. 37, 256-268.

Rigby, B. J., Hirai, N., Spikes, J. D. and Eyring, H. (1959) The mechanical properties of rat tail tendon. $J$. gen. Physiol. 43, 265-283.

VanBrocklin, J. D. and Ellis, D. G. (1965) A study of the mechanical behavior of toe extensor tendons under applied stress. Archs phys. Med. 46, 369-373.

Walker, L. B., Harris, E. H. and Benedict, J. V. (1964) Stress-strain relationship in human cadaveric plantaris tendon: A preliminary study. Med. Electron. biol. Engng 2, 31-38.

Wright, D. G. and Rennels, D. C. (1964) A study of the elastic properties of plantar fascia. J. Bone Jt Surg. 46A, $482-492$. 